\title{
Label-free glucose biosensor based on enzymatic graphene oxide-functionalized tilted fiber grating
}

\author{
Biqiang Jiang ${ }^{a, b, *}$, Kaiming Zhou ${ }^{\text {b,c }}$, Changle Wang ${ }^{\mathrm{b}}$, Qizhen Sun ${ }^{\mathrm{b}, \mathrm{d}}$, Guolu Yin ${ }^{\mathrm{b}}$, \\ Zhijun Tai ${ }^{\mathrm{e}}$, Karen Wilson ${ }^{\mathrm{e}}$, Jianlin Zhao ${ }^{\mathrm{a}}$, Lin Zhang ${ }^{\mathrm{b}, *}$ \\ ${ }^{a}$ MOE Key Laboratory of Space Applied Physics and Chemistry and Shaanxi Key Laboratory of Optical \\ Information Technology, School of Science, Northwestern Polytechnical University, Xi'an 710072, China \\ ${ }^{\mathrm{b}}$ Aston Institute of Photonic Technologies, Aston University, Birmingham B4 7ET, United Kingdom \\ ' State Key Laboratory of Transient Optics and Photonics, Xi'an Institute of Optics and Precision Mechanics, \\ Chinese Academy of Sciences, Xi'an 710119, China \\ ${ }^{\mathrm{d}}$ School of Optical and Electronic Information, National Engineering Laboratory for Next Generation Internet \\ Access System, Huazhong University of Science and Technology, Wuhan 430074, Hubei, China. \\ ${ }^{\mathrm{e}}$ European Bioenergy Research Institute, Aston University, Birmingham B4 7ET, United Kingdom \\ *Corresponding authors. Email address: bqjiang@nwpu.edu.cn (B. Jiang); 1.zhang@aston.ac.uk (L. Zhang)
}

\begin{abstract}
A label-free biosensor based on graphene oxide (GO) and glucose oxidase (GOD) functionalized tilted fiber grating (TFG) with large tilted angle is proposed for low concentration glucose detection. Taking advantages of sufficient binding sites of the GO with oxygen-containing groups, the enzymes (GOD) are covalently immobilized onto GO-deposited TFG via 1-ethyl-3-(3-dimethylaminopropyl) carbodiimide and N-hydroxyl succinimide cross-liner. Surface characterizations with optical microscopy, scanning electron microscopy, Raman and infrared spectroscopy provide detailed assessments and evidences about the homogeneity of GO deposition and the effectiveness of enzyme modification. Through the specific catalysis reaction of GOD on the glucose, a considerable refractive index change in local microenvironment around the TFG results in the resonant wavelength shifts of cladding modes. The detection results of the low-concentration glucose demonstrate that the resonant wavelength has a linear response to the glucose concentration in the range of $0 \sim 8 \mathrm{mM}$ with a response coefficient of $\sim 0.24 \mathrm{~nm} / \mathrm{mM}$, showing an enhanced sensitivity and bio-selectivity compared with the pristine TFG. The miniaturized size and remote label-free sensing capacity of the proposed device permit a multitude of opportunities for single-point measurement in harsh conditions and hard-to-reach spaces, presenting a promising candidate for label-free glucose detection for disease diagnosis, pharmaceutical research and bioengineering applications.
\end{abstract}

Keywords: Fiber grating; optical fiber; glucose; biosensor; graphene oxide; glucose oxidase. 


\section{Introduction}

The rapid and accurate detection of the glucose concentration is critically important in numerous fields ranging from medical diagnosis to pharmaceutical research and quality monitoring in food industry [1]. In particular, the examination of extremely dilute glucose concentration such as the blood glucose level is well worth monitoring and investigating for human's health $[2,3]$. Graphene oxide (GO), one specific branch of graphene research, consists of a single-layer graphite oxide, is considered as a very promising material for biosensors due to its high surface-to-volume ratio, low-cost fabrication and excellent biocompatibility. Compared with the pristine graphene, the presence of various oxygen-containing functional groups in the GO sheet, such as carboxyl groups (-COOH), hydroxyl groups (-OH), epoxide groups (-O-), not only makes it hydrophilic and disperse in water very well, but also serves as sites for chemical modification and further functionalization, thereby provides a remarkable platform for immobilization [4-6]. The intrinsic ability of GO to immobilize some enzymes such as glucose oxidase (GOD) and uricase has instigated researchers' interest to utilize it as an electrochemical biosensor [5, 7-9].

Compared with the electrochemical method, the use of fiber-optic devices as bio/chemical sensing element presents many distinguished advantages for in-situ label-free methods in terms of the overall reduction in costs, size and ease of fabrication [10, 11]. So recently, optical fiber-based biosensors have attracted increasing attention and provided a new approach to diverse detection of bio-parameters [12]. Actually, ordinary single-mode fibers cannot be used directly for biological sensing due to the confinement of optical field by the relatively thick cladding. To pull the guided mode out into the cladding for interaction with the surroundings, many geometry-modified and microstructure fibers as alternatives enable the optical mode to interact with the surroundings, such as side-polished/D-shaped [13, 14], U-shaped [15], tapered fibers [16], photonic crystal fibers (PCF) [17] and grating-assisted fibers mainly including long-period grating (LPG) [18] and tilted fiber grating (TFG) [19, 20]. For biosensing, the implement of surface plasmon resonance (SPR) effects $[10,19,21]$ and bio-selective or enzymatic coating $[18,22,23]$ into the fiber-based sensor as two main approaches are used to further improve the sensitivity of bio-related analytes in lower concentrations. Unlike geometry-modified fibers which need to remove the fiber cladding entirely or in part, grating-assisted fibers retaining a robust mechanical structure, especially TFG, have been used for label-free biomolecule monitoring [19, 24], cellular detection [25], etc. In the previous work of our group, an "excessively tilted" fiber grating (Ex-TFG) structure, breaking the azimuthal symmetry and inducing the strong evanescent field, was developed to excite the polarization-dependent cladding modes and eliminate the cross-sensitive property between the desired measurand and other parameters, particularly temperature and bend [26-28].

In this reported work, the advantages of three components, the intrinsic sensitive performance of TFG with the large tilted angle, the merits of GO biocompatibility and catalysis of enzyme are 
combined to implement a biosensor for improving the glucose detection of low concentration. The TFG, fabricated by UV inscription and scanning phase-mask technique, shows a high sensitivity to surrounding medium' refractive index (SRI), owing to an enhanced evanescent field of high-order cladding mode in the fiber cladding. Furthermore, the GO sheets are deposited on the TFG surface to form a functional layer, allowing the carboxyl groups of GO layer covalently connect with the amino groups of the GOD via the cross-linker of 1-ethyl-3-(3-dimethylaminopropyl) carbodiimide (EDC) and N-hydroxyl succinimide (NHS) [7]. The GOD can be utilized for the enzymatic determination of D-glucose in solution. In the measurement, the catalytic reaction of GOD makes the glucose convert to D-gluconic acid and hydrogen peroxide, leading to the change of the refractive index in the local microenvironment and detectable spectral shift of enzymatic TFG probe. We evaluate the performance of the device by immersing it into a series of glucose solutions with different concentrations, and the detection process is precisely controlled with a micro-fluidic tube and a pipette which allows the measurement of the same amount of the bio-sample solution. The proposed scheme is a valuable approach to remote label-free monitoring of glucose, and expected to be applied in the bio-sensing and medical diagnosis fields.

\section{Fabrication of sensing element and experimental system}

2.1 Materials

Glucose oxidase (GOD, from Aspergillus niger), 1-ethyl-3-(3-dimethylaminopropyl) carbodiimide (EDC), N-hydroxyl succinimide (NHS), $\beta$-D-glucose, phosphate buffered saline (PBS, pH 7.4), sodium chloride $(\mathrm{NaCl})$, sodium hydroxide $(\mathrm{NaOH})$ and nitric acid $\left(\mathrm{HNO}_{3}\right)$ were purchased from Sigma-Aldrich (United Kingdom). BupH MES Buffered Saline (MES, pH 4.7) was purchased from Thermo Fisher Scientific (United Kingdom). Graphene oxide (GO) was purchased from Nanjing XFNANO Materials Tech Co., Ltd, China.

\subsection{Fabrication of TFG}

The TFGs with a large tilted angle of $82^{\circ}$ and a length of $12 \mathrm{~mm}$ were fabricated in hydrogen-loaded (for enhancing the photosensitivity of fiber core) standard single mode fiber (SMF-28, Corning) by using the scanning phase-mask technique and a frequency doubled continuous wave $(\mathrm{CW})$ argon-ion laser with the wavelength of $244 \mathrm{~nm}[19,29]$. The UV laser beam was focused on the optical fiber by a cylindrical lens and the grating (periodic modulation of refractive index) was imprinted in the photosensitive core by using a phase mask placed in front of the fiber. The phase mask was slanted with respect to the fiber axis, with the angle as a key factor to determine the excited cladding modes and sensing capacity to the surroundings. After UV inscription, the TFGs were annealed at $80{ }^{\circ} \mathrm{C}$ for 48 hours to stabilize and remove of residual hydrogen.

\subsection{Surface functionalization of TFG}

To enable selectivity of TFG for specific bio-sample such as glucose, it is necessary to functionalize the fiber surface. Figure 1 schematically depicts the key functionalization process of 
the optical fiber biosensor, including the deposition of GO and the immobilization of GOD on the fiber surface.

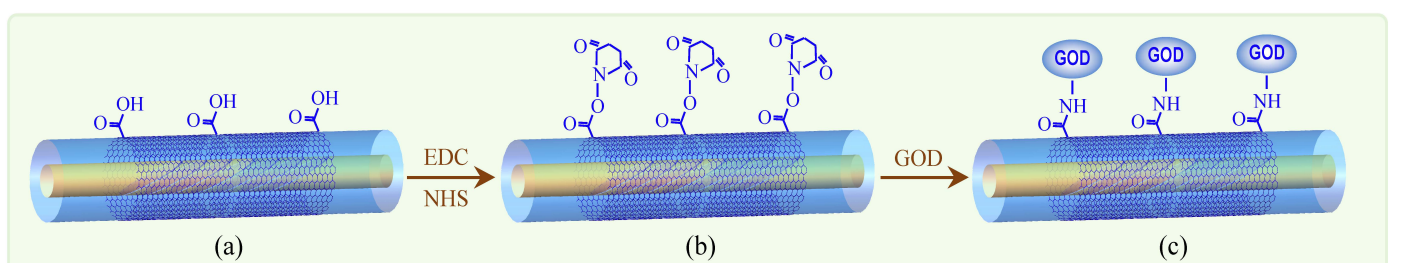

Fig. 1. Schematic diagram of GOD immobilization on GO using EDC/NHS as cross-linkers. (a) GO-coated, (b) EDC/NHS-treated and (c) GOD-immobilized TFG.

The fabricated TFG was firstly soaked in $\mathrm{HNO}_{3}$ solution $(5 \% \mathrm{v} / \mathrm{v})$ for $1 \mathrm{~h}$ at room temperature to remove the contaminant and then thoroughly rinsed with deionized (DI) water and ethanol for several times. The exfoliated suspension of GO was prepared through dispersing GO powder in DI water by ultrasonic treatment, and then we obtained a homogeneous and golden-brown suspension with the concentration of $1 \mathrm{mg} / \mathrm{ml}$. Then, the GO suspension was deposited on the surface of the TFG using a dip-coating technique [15]. For assisting GO deposition on the fiber surface, the TFG region was kept on a $50^{\circ} \mathrm{C}$ heating platform and its two ends were clamped with two stages to keep the fiber straight. The transmission spectrum was monitored in real-time with an optical spectrum analyzer (OSA). After dropping the GO suspension three times and keeping the grating region immersed in the solution for $\sim 30 \mathrm{~min}$ each time, the coupling intensity of cladding mode in the spectrum decreased by $\sim 8 \mathrm{~dB}$. Afterwards, the GO-coated TFG was rinsed with the DI water to remove the floating GO sheets and tested for the stability.

After GO deposition, the chemical attachment of GO and GOD was carried out with the help of the mixture of two cross-linking reagents of EDC and NHS. The GO-coated TFG was incubated in the activation buffered solution ( $0.1 \mathrm{M} \mathrm{MES,}, 0.5 \mathrm{M} \mathrm{NaCl}, \mathrm{pH}$ 6.0) containing $15 \mathrm{mM}$ EDC and $30 \mathrm{mM}$ NHS for $1 \mathrm{~h}$ to activate the carboxyl groups in GO layer around the fiber, as depicted in Figs. 1(a) and 1(b). GOD was subsequently immobilized on the GO coating by incubating the fiber in the coupling buffered solution (10 mg/ml GOD/PBS) for $1 \mathrm{~h}$ at room temperature. And then, the GO-coated TFG with GOD immobilization was thoroughly rinsed with PBS buffered solution and DI water and dried in air. As shown in Fig. 1(c), with its amino groups of GOD covalently attached to the carboxyl groups of GO, the enzyme GOD was immobilized around the surface of the TFG, and then the fabricated GOD-GO-modified TFG as sensing element is used to detect the glucose sample.

\subsection{Experimental system}

We examine the sensing performance of fabricated device by using the experimental system schematically depicted in Fig. 2. Launched from a broadband light source (BBS), the broadband light covering several hundreds of nanometers $(1200 \sim 1700 \mathrm{~nm})$ was transmitted into the TFG probe via a fiber polarizer and polarization controller (PC) and monitored using the OSA with a 
minimum wavelength resolution of $0.02 \mathrm{~nm}$. The polarization state of the input light is adjusted with the linear polarizer and PC. During the experiments, the TFG was fixed at the two far ends to avoid the axial strain and bending. A micro-fluidic tube of a diameter of $1.5 \mathrm{~mm}$, a height of $1 \mathrm{~mm}$ and a length of $20 \mathrm{~mm}$, as the sample cell, was fixed on a lifting stage for controlling of its position. In order to eliminate the difference caused by the amount and shape of the sample solution, for each measurement, the same amount of sample $(30 \mu \mathrm{L})$ was injected into the sample cell with a pipette.

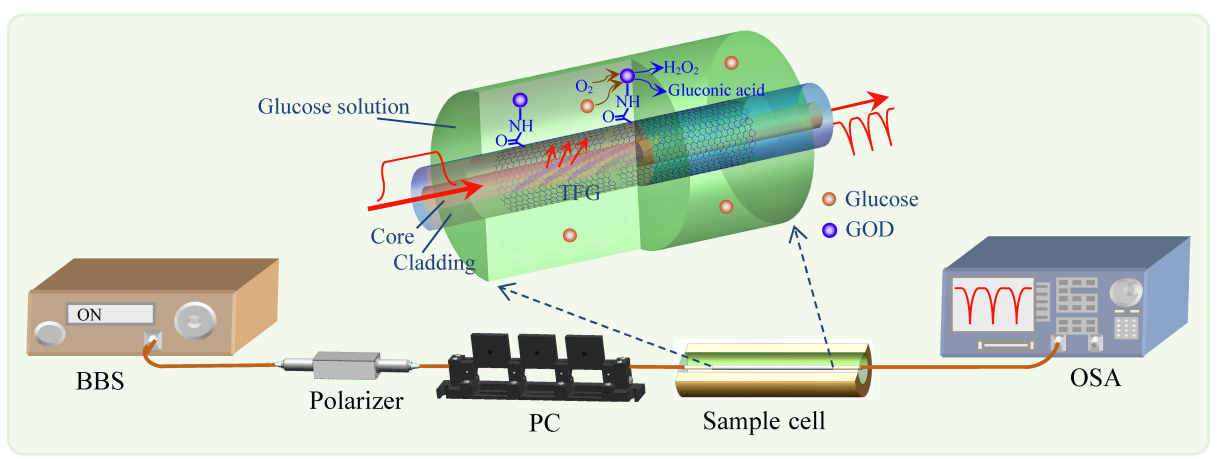

Fig. 2. Experimental setup for surrounding medium and glucose detection. The upward inset shows the schematically dissection diagram of GOD-GO-modified TFG. BBS: broadband source; PC: polarization controller; OSA: optical spectral analyzer.

\section{Results and discussion}

\subsection{Optical properties of fabricated TFG and SRI response}

Figure 3(a) shows the microscopic image of $82^{\circ}$ tilted fringes and $27.38 \mu \mathrm{m}$ grating period in the fiber core by a high-resolution microscope (Carl Zeiss Axiotron 2). The grating planes of the TFG greatly enhance the light coupling from the forward-propagating core mode into the forward-propagating cladding modes according to the phase matching condition [29]. And hence, a series of cladding mode resonances appear at discrete wavelengths, and each resonance has two orthogonal TE and TM polarization states. The full spectrum in the wavelength region of 1330 1700 nm is shown in Fig. 3(b) for un-polarized light (with removal of the polarizer and polarization controller from the system in Fig. 2), exhibiting the dual-peak feature of the cladding modes. It can also be obtained that with increasing the resonant wavelength (or mode order), the separation of adjacent cladding modes increases from $\sim 50 \mathrm{~nm}$ to $\sim 64 \mathrm{~nm}$, and the wavelength difference of dual-peak increases from $4.6 \mathrm{~nm}$ to $6.9 \mathrm{~nm}$, thus the dual-peak feature is mode order-dependent. For a specific order of cladding mode such as the peak at around 1560nm, when the linearly polarized light was launched into the TFG, the resonance peaks of two orthogonal polarizations are obtained by adjusting the PC, which are related to TM and TE cladding modes, as shown in the inset of Fig. 3(b).

According to the phase matching condition, for the TFG with the large tilted angle, the resonant wavelength $\lambda_{i, \text { clad }}^{m}$ of the $m$ th cladding mode can be expressed as follows [29] 


$$
\lambda_{i, \text { clad }}^{m}=\left[n_{\text {eff, core }}-n_{i, \text { eff, clad }}^{m}\left(n_{\mathrm{SRI}}\right)\right] \Lambda_{\mathrm{g}} / \cos \theta,
$$

where, $i$ represents TE or TM, $\Lambda_{\mathrm{g}}$ is the nominal grating period, $\theta$ is the tilt angle, $n_{\text {eff,core }}$ and $n_{i, \text { eff,clad }}^{m}$ are the effective indices of the core mode and the $m$ th cladding mode, respectively. Also from Eq. (1), with grating parameters $n_{\text {eff,core }}, \Lambda_{\mathrm{g}}$ and $\theta$ known, the resonant wavelength of the cladding mode is determined by its effective index which is depended on the SRI, and this is the fundamental principle for measurement of the SRI or bio-detection.
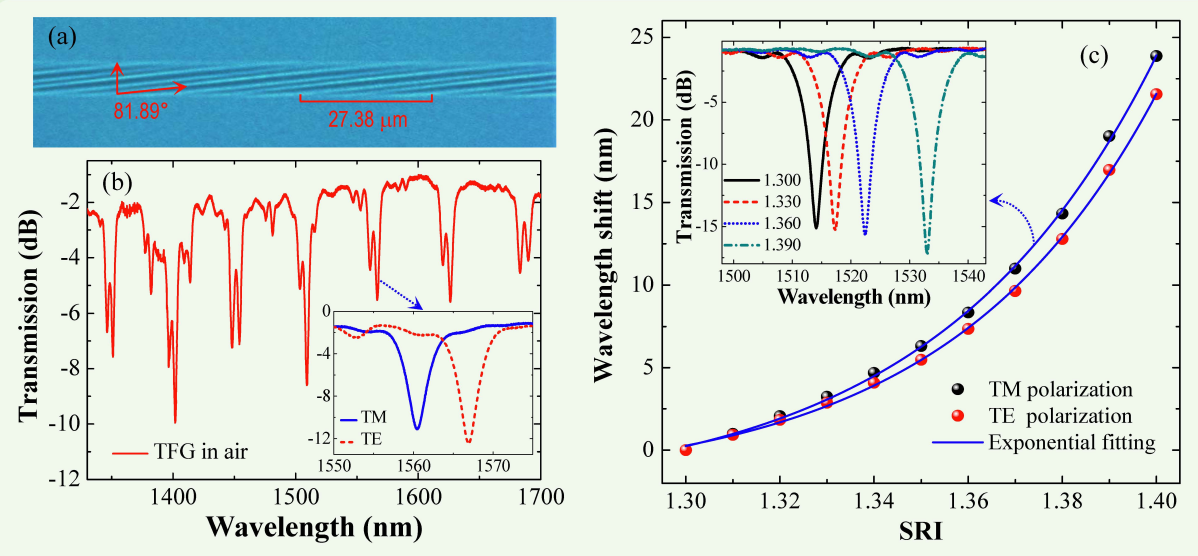

Fig. 3. (a) Optical microscopic image of tilted fringes of the TFG; (b) its transmission spectrum in the region of 1330 1700 nm for un-polarized light, the inset shows the dual-peak feature in the zoomed region of $1550 \sim 1580 \mathrm{~nm}$ when the TFG was probed with two orthogonally polarized lights; (c) SRI response of resonant wavelengths at TM and TE cladding modes, the inset shows the spectral evolution of TM mode.

We firstly examined the SRI response of the fabricated TFG by using a series of refractive index liquids (from Cargille Labs) ranging from 1.300 to 1.400 with an interval of 0.01 . The results are shown in Fig. 3(c). Since the spectral variation including the resonant wavelength and peak intensity has similar manner for the two peaks [30], we present the spectral evolution of TM peak with SRI, as depicted in the inset of Fig. 3(c). We can also observe a small increase of the peak intensity caused by the SRI-induced variation of coupling coefficient. From the response curves of two polarizations with SRI, the resonant wavelengths demonstrate the exponential red-shift trends with the increasing SRI in the range of 1.300 1.400. According to the exponential fitting relationships, the calculated SRI sensitivities of TM and TE modes are $127.53 \mathrm{~nm} / \mathrm{RIU}$ and $\sim 110.10 \mathrm{~nm} / \mathrm{RIU}$ (RIU stands for "refractive index unit") near the SRI of 1.33, respectively. In the experiment described below, with the consideration of relatively higher sensitivity in TM polarization, we only employed the TM resonance for the glucose detection.

\subsection{Surface characterizations and spectroscopic investigation of the GOD-GO-modified TFG}

After the surface modification and functionalization, the GOD-GO-modified TFG is firstly characterized by the aforementioned high-resolution microscope. Figure 4 displays the microscope 
images of the device at different stages in the surface modification process: original fiber, GO-coated, EDC/NHS-treated and GOD-immobilized. It can be observed that after deposition of yellow-brown wrinkled GO film, there is no noticeable difference except gradually deepened color. However, by observing the fluorescence excited with a laser of $430 \mathrm{~nm}$, we found obvious difference for these stages: the fluorescence firstly appeared with GO coating (see the inset of Fig. 4(b)), which originates from electronic coupling of carboxylic acid groups with nearby carbon atoms of graphene [31, 32], then disappeared after EDC/NHS-treatment due to the reduction of the carboxylic acid groups, but re-appeared in the presence of GOD molecules [18] (see the inset of Fig. 4(d)), and the fluorescence of GO coating is much stronger than that after GOD immobilization. To a certain extent, the change of fluorescence is attributed to different treatments on the TFG surface. In the meantime, we observed the change of the radiation field near around the TFG region with a $635 \mathrm{~nm}$ laser. For the bare TFG, due to the fact that the light is the cladding guided and hence propagates as a bound surface wave along the cladding, thus we cannot observe the bright line in the grating region by a far field camera. However, after coating, there appears the bright scattered line, as shown in Fig. 4(e), which demonstrates that the leaky modes radiated from the cladding are key signatures of strong enhancement of evanescent field by GO in terms of coupling, absorption and scattering. The surface of GO-coated TFG was also examined by scanning electron microscopy (SEM) and shown in Figs. 4(f) and 4(g), and a wrinkled but relative uniform GO film wraps around the fiber tightly and completely under high magnification. From the fiber cross-section shown in the inset of Fig. 4(g), the thickness of GO layer is approximately $\sim 300 \mathrm{~nm}$. At the macro level, $\sim 12 \mathrm{~mm}$ length of GO layer shown in Fig. 4(e) can cover the whole grating region and provide an appropriate matrix for further GOD immobilization.

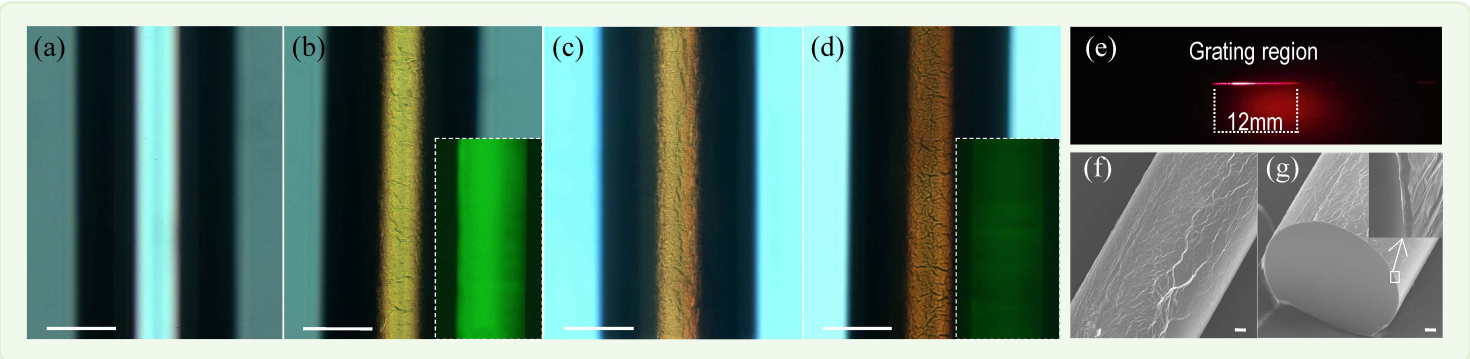

Fig. 4. Optical microscope images of (a) original, (b) GO-coated, (c) EDC/NHS-treated and (d) GOD-immobilized TFG, the insets of (b) and (d) show the corresponding fluorescence images; (e) radiation field of the GO-coated TFG when launched by a $635 \mathrm{~nm}$ laser; (f) and (g) SEM images of different regions of GOD-GO-modified TFG, the inset of ( $g$ ) show the zoomed section of the device. Scale bar: (a-d) $125 \mu \mathrm{m}$; (f and g) $10 \mu \mathrm{m}$.

Due to unique Raman scattering properties of GO, we further characterized the bare and GO-coated fibers by measuring the Raman spectra to verify the efficiency of GO coating, as shown in Fig. 5(a). In comparison to the spectrum of the bare fiber, the prominent bands can be observed around 1354 and $1597 \mathrm{~cm}^{-1}$ (Fig. 5(a)), corresponding to the D and G Raman vibrational modes of graphitic carbon sheet, respectively [33]. The G band reflects the first-order scattering of 
the in-plane optical phonon $\mathrm{E}_{2 \mathrm{~g}}$ mode, and the high intensity (relative to the $\mathrm{G}$ band) of $\mathrm{D}$ band reflects the ring vibration symmetrical breathing mode and associates with the defects caused by the attachment of hydroxyl and epoxide groups [15]. Meanwhile, we choose the region shown in Fig. 5(b) to acquire the Raman mapping of GO-coated fiber by integrating the peak intensity at the D-band. From the Raman mapping in Fig. 5(c), it can be seen that a clear edge and the relative uniform distribution of GO coating around the fiber.
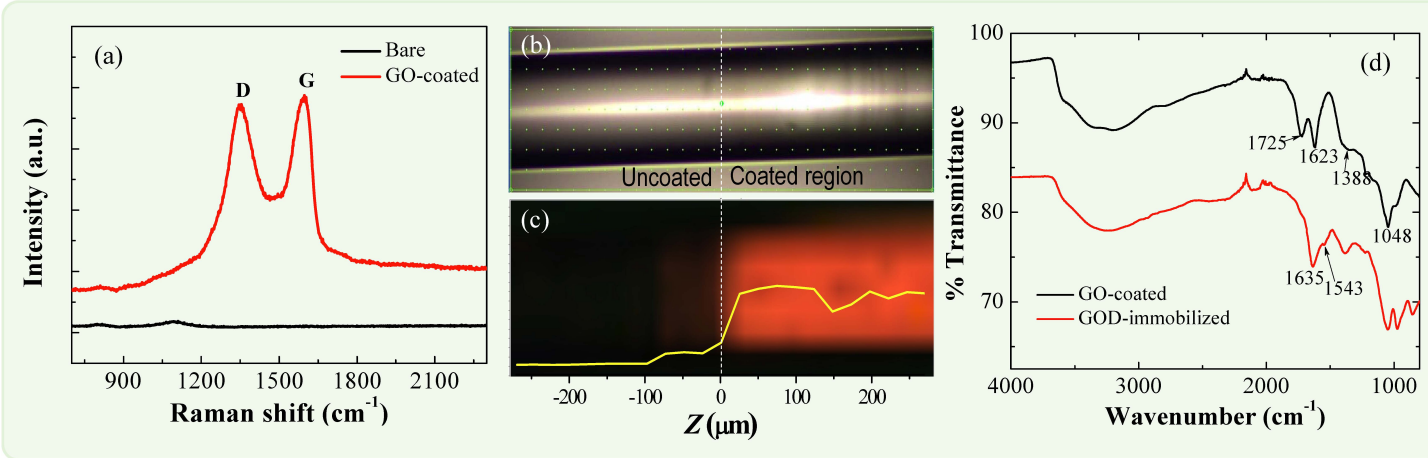

Fig. 5. (a) Raman spectra of bare and GO-coated fiber showing the G and D bands of GO; (b) fiber region near the GO coating edge and the corresponding confocal Raman mapping at the D-band, and 1-dimension Raman distribution (yellow curve) along the central axis of the fiber; (d) ATR-IR spectra of GO-coated and GOD-immobilized optical fibers.

To obtain complementary information on various qualitative bonds and functional groups and then validate the efficiency of GO deposition and subsequent GOD grafting process, the attenuated total reflectance-infrared (ATR-IR) spectra were recorded by using an ATR-IR spectrometer (Thermo Scientific Nicolet iS50 in the range of 4000 600 $\mathrm{cm}^{-1}$ ), as shown in Fig. 5(d). From the ATR-IR spectrum of GO, the appearances of the intense bands at 1725, 1623, 1388 and $1048 \mathrm{~cm}^{-1}$ were attributed to the stretching vibrations of $\mathrm{C}=\mathrm{O}, \mathrm{C}=\mathrm{C}, \mathrm{C}-\mathrm{O}$ and $\mathrm{C}-\mathrm{O}-\mathrm{C}$ groups, respectively [34, 35], indicating the existence of a certain amount of oxygen-containing groups. From the spectrum after GOD immobilization, we can find that the peaks of GO still existed, but two new peaks at 1635 and $1543 \mathrm{~cm}^{-1}$ appeared, depicting the bending vibrations of the amide I and II groups $[18,35,36]$. Thus, the above analysis of ATR-IR spectra difference revealed that the GOD was effectively covalent-immobilized on the GO-coated TFG surface.

\subsection{Glucose detection with the GOD-GO-modified TFG}

Firstly, the spectral responses before and after GO and GOD functionalization were monitored by the setup of Fig. 2 when the TFG was immersed in the background solution (DI water). The results are shown in the inset of Fig. 6(a). Clearly, the significant spectrum shift and attenuation as a result of the interaction between the evanescent field and the functionalized coating with a complex refractive index [37, 38]. The resonant wavelength red-shifted by $8.85 \mathrm{~nm}$ due to the high refractive index of the coating. Also, the absorption and scattering of the coating results in a noticeable decrease of the resonant peak. 
We prepared a series of dilute $\beta$-D-glucose solutions with different concentrations ranging from $1 \mathrm{mM}$ to $10 \mathrm{mM}$ with interval of $1 \mathrm{mM}$ and used the experimental setup depicted in Fig. 2 to examine the glucose sensing performance of GOD-GO-modified TFG. As a comparison, we firstly tested the response of a bare TFG. Due to the lack of enzyme catalysis, the spectrum almost remained unchanged in the extremely small concentration (or SRI) change of glucose solutions from $0 \mathrm{mM}$ to $10 \mathrm{mM}$, as shown in Fig. 6(a). However, for the GOD-GO-modified TFG, due to the high specificity and selectivity of the enzymes for $\beta$-D-glucose, the GOD can catalyze the oxidation of glucose in the following reaction,

$$
\beta \text { - D - Glucose }+\mathrm{H}_{2} \mathrm{O}+\mathrm{O}_{2} \stackrel{\text { GOD }}{\longrightarrow} \mathrm{D} \text { - Gluconic Acid }+\mathrm{H}_{2} \mathrm{O}_{2} \text {. }
$$

When the glucose solution was filled into the sample cell, the $\beta$-D-glucose was converted to D-glucono-1,5-lactone and hydrogen peroxide, and finally the former was hydrolyzed to D-gluconic acid. And then, comparing with the TFG without functionalization, a considerable shift of the resonant wavelength of the cladding mode caused by a relatively larger change of SRI in the local microenvironment causing was observed. That is to say, by exploiting the catalytic reaction of the enzyme, the sensitivity and bio-selectivity of the glucose detection can be effectively improved. The glucose solutions of different concentrations $(1,2,3, \ldots, 10 \mathrm{mM})$ were introduced in the sample cell in turn, the TFG probe was subsequently immersed into the solutions, and then the corresponding spectra were recorded using the OSA. After each measurement, the TFG was rinsed with DI water to remove the water-soluble reactants (glucose) and products (gluconic acid and hydrogen peroxide), making the spectrum revert to the original one in the water. During the experiment, the temperature was kept constant at $\sim 20^{\circ} \mathrm{C}$ to rule out any uncertainty caused by the temperature fluctuation. Figure 6(b) displays the evolution of TFG transmission spectra against the glucose concentration, and we observe a significant red-shift $(\sim 2 \mathrm{~nm})$ of resonant wavelength and peak intensity attenuation.
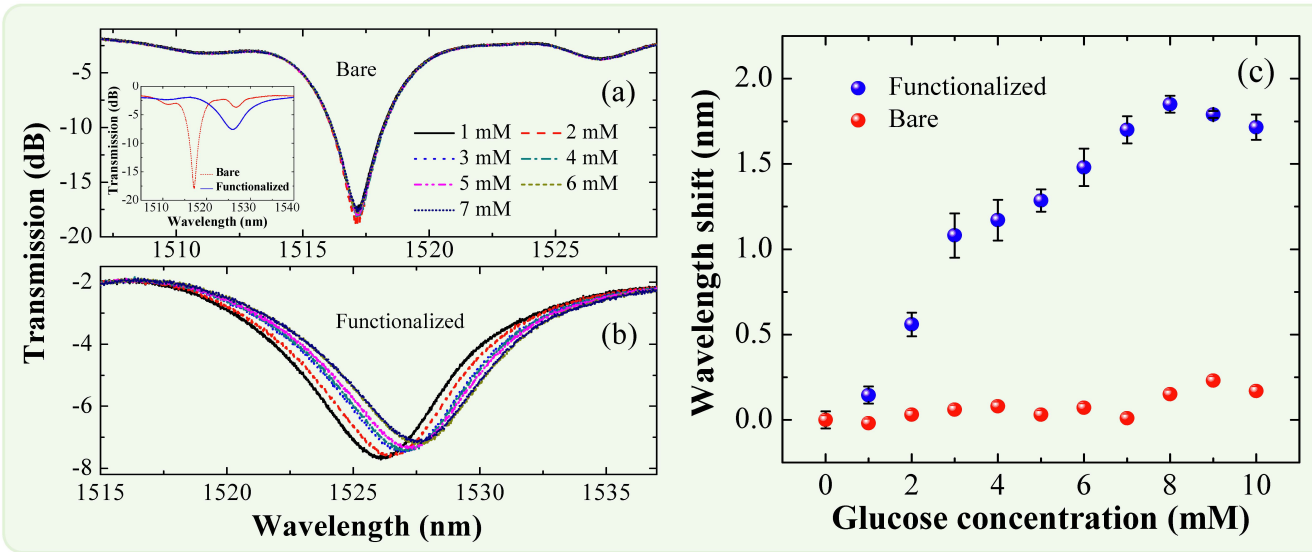

Fig. 6. Spectral evolution of the TFG (a) before and (b) after GO and GOD functionalization, the inset shows the spectral change in water before and after functionalization, and (c) corresponding resonant wavelength shift with the glucose concentration.

In more detail, to clearly demonstrate the effect of the GOD and GO functionalization on the 
glucose detection, Fig. 6(c) presents the wavelength shift of the cladding mode resonance with the glucose concentration. In the lower concentration range of $0 \sim 8 \mathrm{mM}$, the GOD-GO-modified TFG exhibits a linear response of resonant wavelength versus concentration, and the measured sensitivity is $\sim 0.24 \mathrm{~nm} / \mathrm{mM}$ with an $R$-square value 0.97 from the linear fitting result. The wavelength shift of the glucose sensor is closely related to the coverage area and the amount of the active GOD molecules. Therefore, considering the length and the diameter are only $12 \mathrm{~mm}$ and $125 \mu \mathrm{m}$, respectively, when the concentration is higher than $8 \mathrm{mM}$, the wavelength shift arrives at a plateau, which can be explained by the exhaustion of the entire functionalized surface by glucose and then the saturation by gluconic acid, leading to a stable microenvironment and thus its refractive index will not change and even slightly decrease with the increase of the glucose concentration. However, for the unmodified sample, the measurement results from Fig. 6(c) show the resonant wavelength has almost no correlation with the glucose concentration, and the maximum wavelength fluctuation is only $\sim 0.23 \mathrm{~nm}$. From the comparing results between the unmodified and modified TFG, the glucose detection sensitivity is greatly improved in the range of $0 \sim 8 \mathrm{mM}$, and thus the functionalization treatment based on $\mathrm{GO}$ coating with excellent biocompatibility and GOD immobilization with catalytic effect is essential and effective. In addition, the reusability is the key factor of the sensor for the practical applications, therefore, the second cycle of glucose measurements were carried out under the same conditions. The vertical error bars at each point in Fig. 6(c) indicate the maximum differences relative to the mean value of two measurements. From the results of multiple points and two cycles of experiment measurements, the proposed device is possible to be used for multiple times.

Table 1 gives the sensing performance in comparison with the previous reports based on LPG [18] and $81^{\circ}$-TFG in SMF-28 [23]. Disclosed by the narrower bandwidth of resonant peaks, the TFGs have higher Q-factor than LPG. In the proposed device, the glucose doesn't need to be dissolved in sodium acetate (SA) buffered solution for higher RI $(\sim 1.37)$ and lower $\mathrm{pH}$ value $(\sim 5.2)$, but only in DI water which has the similar RI and $\mathrm{pH}$ values with body fluid. More importantly, through the unit conversion with the molecular weight $180.16 \mathrm{~g} / \mathrm{mol}$ of $\beta$-D-glucose, the sensitivity of our device is around $1.33 \mathrm{~nm} /(\mathrm{mg} / \mathrm{ml})$, which is $\sim 4.5$ and $\sim 1.7$ times higher than of the $81^{\circ}$-TFG without GO layer and LPG based glucose biosensors [18, 23]. And also, the analytical range of $0 \sim 8 \mathrm{mM}$ covers the important biological region for human blood glucose levels between 3.90 5.59 $\mathrm{mM}$. Therefore, due to the inherently high surface-to-volume ratio and abundant oxygen-containing functional groups, the presence of GO layer as a good matrix provides plenty of binding sites and excellent biocompatibility, and then can help the Ex-TFG to enhance the sensitivity in glucose measurement without special buffered solution required.

Table 1. Comparison of sensing performance between different fiber grating-based glucose sensors

\begin{tabular}{|c|c|c|c|c|c|c|}
\hline $\begin{array}{l}\text { Sensing } \\
\text { element }\end{array}$ & $\begin{array}{c}\text { Length } \\
(\mathrm{mm})\end{array}$ & $\begin{array}{c}\text { SRI sensitivity } \\
\text { (nm/RIU) }\end{array}$ & Q-factor & $\begin{array}{c}\text { Buffered } \\
\text { solution }\end{array}$ & $\begin{array}{c}\text { Glucose } \\
\text { sensitivity }\end{array}$ & $\begin{array}{c}\text { Measurement } \\
\text { range }\end{array}$ \\
\hline
\end{tabular}




\begin{tabular}{|c|c|c|c|c|c|c|}
\hline $\begin{array}{l}\text { LPG (with } \\
\text { APTES) }\end{array}$ & 20 & $-50 \sim-100$ & $\sim 80$ & $\begin{array}{c}\text { Sodium acetate } \\
\text { (SA) }\end{array}$ & $\begin{array}{c}0.806 \\
\mathrm{~nm} /(\mathrm{mg} / \mathrm{ml})\end{array}$ & $0.1 \sim 3.0 \mathrm{mg} / \mathrm{ml}$ \\
\hline $\begin{array}{c}\text { 81ํ-TFG (with } \\
\text { APTES) }\end{array}$ & 10 & $\sim 168$ & $\sim 352$ & $\begin{array}{l}\text { Sodium acetate } \\
\text { (SA) }\end{array}$ & $0.298 \mathrm{~nm} /(\mathrm{mg} / \mathrm{ml})$ & $0.0 \sim 3.0 \mathrm{mg} / \mathrm{ml}$ \\
\hline $\begin{array}{c}\text { GO-modified } \\
\text { TFG }\end{array}$ & 12 & $\sim 128$ & $\sim 339$ & DI Water & $\begin{array}{c}0.24 \mathrm{~nm} / \mathrm{mM} \\
\approx 1.33 \mathrm{~nm} /(\mathrm{mg} / \mathrm{ml})\end{array}$ & $\begin{array}{c}0 \sim 8 \mathrm{mM} \\
(0.0 \sim 1.5 \mathrm{mg} / \mathrm{ml})\end{array}$ \\
\hline
\end{tabular}

\section{Conclusions}

We have implemented a lab-on-fiber device based on an GOD-GO-modified TFG to achieve the label-free and selective glucose detection in the low concentration level. The TFG with large tilted angle has been demonstrated to sense the concentration change of the glucose solution in which the catalytic reaction of enzyme occurred. The GO sheets with abundant binding sites were firstly deposited onto the TFG surface to provide a favorable platform for enzyme immobilization, and then the EDC and NHS as the cross-linking reagents help to form the linkage between carboxyl groups of GO and the amine of GOD. The investigations of optical microscopy, SEM, Raman spectroscopy and ATR-IR show the relatively uniform coating of GO and effective GOD immobilization. Also, in the presence of functionalization coating, the coupling intensity of TFG cladding mode has a decrease due to the interaction between the optical mode and coating materials. By monitoring the resonant wavelength shift with the glucose concentration, the GOD-GO-modified TFG shows a linear response with the sensitivity of $\sim 0.25 \mathrm{~nm} / \mathrm{mM}$ in the low glucose concentration range of $0 \sim 8 \mathrm{mM}$. In addition, the covalent linking method originated from electrochemistry is employed in optical fiber sensor for glucose detection, which combines the advantages of the electrochemical and optical fiber sensing techniques, such as high-sensitive, remote, label-free and in-situ measurement, and provides a reference or inspiration for other bio-sensors. Therefore, the proposed enzymatic GO-based fiber-optic biosensor is a promising alternative and expected to be applied in medicine, chemical monitoring and food industry.

\section{Acknowledgement}

This work was funded by the Marie Skłodowska-Curie Individual Fellowships in the European Union's Horizon 2020 Research and Innovation Programme (660648), the National Natural Science Foundation of China (NSFC) $(61505165,61405128)$, and the Natural Science Basic Research Plan in Shaanxi Province of China (2016JQ6032). The authors thank Dr Zhaoliang Zheng and Jianli Zou (Stephenson Institute for Renewable Energy and Department of Chemistry, University of Liverpool) for fruitful discussion and assistance with Raman characteristics.

\section{References}

[1] C. Tiangco, D. Fon, N. Sardesai, Y. Kostov, F. Sevilla Iii, G. Rao, et al., Fiber optic biosensor for transdermal glucose based on the glucose binding protein, Sens. Actuators B Chem., 242(2017) 
569-576.

[2] S. Tierney, B.M.H. Falch, D.R. Hjelme, B.T. Stokke, Determination of glucose levels using a functionalized hydrogel-optical fiber biosensor: Toward continuous monitoring of blood glucose in vivo, Anal. Chem., 81(2009) 3630-3636.

[3] J. Wang, Electrochemical glucose biosensors, Chem. Rev., 108(2008) 814-825.

[4] D. Chen, H. Feng, J. Li, Graphene oxide: preparation, functionalization, and electrochemical applications, Chem. Rev., 112(2012) 6027-6053.

[5] T. Kuila, S. Bose, P. Khanra, A.K. Mishra, N.H. Kim, J.H. Lee, Recent advances in graphene-based biosensors, Biosens. Bioelectron., 26(2011) 4637-4648.

[6] Y.V. Stebunov, O.A. Aftenieva, A.V. Arsenin, V.S. Volkov, Highly sensitive and selective sensor chips with graphene-oxide linking layer, ACS Applied Materials \& Interfaces, 7(2015) 21727-21734.

[7] Y. Liu, D. Yu, C. Zeng, Z. Miao, L. Dai, Biocompatible graphene oxide-based glucose biosensors, Langmuir, 26(2010) 6158-6160.

[8] Z. Wang, X. Zhou, J. Zhang, F. Boey, H. Zhang, Direct electrochemical reduction of single-layer graphene oxide and subsequent functionalization with glucose oxidase, J. Phys. Chem. C, 113(2009) 14071-14075.

[9] M.N. Omar, A.B. Salleh, H.N. Lim, A. Ahmad Tajudin, Electrochemical detection of uric acid via uricase-immobilized graphene oxide, Anal. Biochem., 509(2016) 135-141.

[10] C. Caucheteur, T. Guo, J. Albert, Review of plasmonic fiber optic biochemical sensors: improving the limit of detection, Anal. Bioanal. Chem., 407(2015) 3883-3897.

[11] T. Guo, F. Liu, B.-O. Guan, J. Albert, [INVITED] Tilted fiber grating mechanical and biochemical sensors, Opt. Laser Technol., 78, Part B(2016) 19-33.

[12] X.D. Wang, O.S. Wolfbeis, Fiber-optic chemical sensors and biosensors (2013-2015), Anal. Chem., 88(2016) 203-227.

[13] Y. Wu, B.C. Yao, A.Q. Zhang, X.L. Cao, Z.G. Wang, Y.J. Rao, et al., Graphene-based D-shaped fiber multicore mode interferometer for chemical gas sensing, Opt. Lett., 39(2014) 6030-6033.

[14] B. Jiang, M. Xue, C. Zhao, D. Mao, K. Zhou, L. Zhang, et al., Refractometer probe based on a reflective carbon nanotube-modified microfiber Bragg grating, Appl. Opt., 55(2016) 7037-7041.

[15] S.S. Gao, H.W. Qiu, C. Zhang, S.Z. Jiang, Z. Li, X.Y. Liu, et al., Absorbance response of a graphene oxide coated U-bent optical fiber sensor for aqueous ethanol detection, RSC Adv., 6(2016) 15808-15815.

[16] H.-Y. Lin, C.-H. Huang, G.-L. Cheng, N.-K. Chen, H.-C. Chui, Tapered optical fiber sensor based on localized surface plasmon resonance, Opt. Express, 20(2012) 21693-21701.

[17] Y.C. Tan, Z.Q. Tou, V. Mamidala, K.K. Chow, C.C. Chan, Continuous refractive index sensing based on carbon-nanotube-deposited photonic crystal fibers, Sens. Actuators B Chem., 202(2014) 1097-1102.

[18] A. Deep, U. Tiwari, P. Kumar, V. Mishra, S.C. Jain, N. Singh, et al., Immobilization of enzyme on long period grating fibers for sensitive glucose detection, Biosens. Bioelectron., 33(2012) 190-195. 
[19] T. Guo, F. Liu, X. Liang, X. Qiu, Y. Huang, C. Xie, et al., Highly sensitive detection of urinary protein variations using tilted fiber grating sensors with plasmonic nanocoatings, Biosens. Bioelectron., 78(2016) 221-228.

[20] B. Jiang, X. Lu, X. Gan, M. Qi, Y. Wang, L. Han, et al., Graphene-coated tilted fiber-Bragg grating for enhanced sensing in low-refractive-index region, Opt. Lett., 40(2015) 3994-3997.

[21] L.-Y. Shao, J.P. Coyle, S.T. Barry, J. Albert, Anomalous permittivity and plasmon resonances of copper nanoparticle conformal coatings on optical fibers [Invited], Opt. Mater. Express, 1(2011) 128-137.

[22] Y. Huang, Z. Tian, L.-P. Sun, D. Sun, J. Li, Y. Ran, et al., High-sensitivity DNA biosensor based on optical fiber taper interferometer coated with conjugated polymer tentacle, Opt. Express, 23(2015) 26962-26968.

[23] B. Luo, Z. Yan, Z. Sun, J. Li, L. Zhang, Novel glucose sensor based on enzyme-immobilized $81^{\circ}$ tilted fiber grating, Opt. Express, 22(2014) 30571-30578.

[24] C. Ribaut, V. Voisin, V. Malachovská, V. Dubois, P. Mégret, R. Wattiez, et al., Small biomolecule immunosensing with plasmonic optical fiber grating sensor, Biosens. Bioelectron., 77(2016) 315-322.

[25] V. Malachovská, C. Ribaut, V. Voisin, M. Surin, P. Leclère, R. Wattiez, et al., Fiber-optic SPR immunosensors tailored to target epithelial cells through membrane receptors, Anal. Chem., $87(2015)$ 5957-5965.

[26] K. Zhou, L. Zhang, X. Chen, I. Bennion, Optic sensors of high refractive-index responsivity and low thermal cross sensitivity that use fiber Bragg gratings of $>80^{\circ}$ tilted structures, Opt. Lett., 31(2006) 1193-1195.

[27] Z. Yan, H. Wang, C. Wang, Z. Sun, G. Yin, K. Zhou, et al., Theoretical and experimental analysis of excessively tilted fiber gratings, Opt. Express, 24(2016) 12107-12115.

[28] Z. Yan, Z. Sun, K. Zhou, B. Luo, J. Li, H. Wang, et al., Numerical and experimental analysis of sensitivity-enhanced RI sensor based on Ex-TFG in thin cladding fiber, J. Lightwave Technol., 33(2015) 3023-3027.

[29] K. Zhou, L. Zhang, X. Chen, I. Bennion, Low thermal sensitivity grating devices based on Ex-45 tilting structure capable of forward-propagating cladding modes coupling, J. Lightwave Technol., 24(2006) 5087-5094.

[30] B. Jiang, G. Yin, K. Zhou, C. Wang, X. Gan, J. Zhao, et al., Graphene-induced unique polarization tuning properties of excessively tilted fiber grating, Opt. Lett., 41(2016) 5450-5453.

[31] J. Shang, L. Ma, J. Li, W. Ai, T. Yu, G.G. Gurzadyan, The origin of fluorescence from graphene oxide, Scientific Reports, 2(2012) 792.

[32] C. Galande, A.D. Mohite, A.V. Naumov, W. Gao, L. Ci, A. Ajayan, et al., Quasi-molecular fluorescence from graphene oxide, Scientific Reports, 1(2011) 85.

[33] K.N. Kudin, B. Ozbas, H.C. Schniepp, R.K. Prud'homme, I.A. Aksay, R. Car, Raman spectra of graphite oxide and functionalized graphene sheets, Nano Lett., 8(2008) 36-41.

[34] W. Chen, L. Yan, P.R. Bangal, Preparation of graphene by the rapid and mild thermal reduction of graphene oxide induced by microwaves, Carbon, 48(2010) 1146-1152. 
[35] L. Zhou, Y. Jiang, J. Gao, X. Zhao, L. Ma, Graphene oxide as a matrix for the immobilization of glucose oxidase, Appl. Biochem. Biotechnol., 168(2012) 1635-1642.

[36] Y.C. Jung, H. Muramatsu, K. Fujisawa, J.H. Kim, T. Hayashi, Y.A. Kim, et al., Optically and biologically active mussel protein-coated double-walled carbon nanotubes, Small, 7(2011) 3292-3297.

[37] I. Jung, M. Vaupel, M. Pelton, R. Piner, D.A. Dikin, S. Stankovich, et al., Characterization of thermally reduced graphene oxide by imaging ellipsometry, J. Phys. Chem. C, 112(2008) 8499-8506.

[38] Sridevi S, K.S. Vasu, N. Jayaraman, S. Asokan, A.K. Sood, Optical bio-sensing devices based on etched fiber Bragg gratings coated with carbon nanotubes and graphene oxide along with a specific dendrimer, Sens. Actuators B Chem., 195(2014) 150-155. 